\title{
Kniest Dysplasia Is Characterized by an Apparent Abnormal Processing of the C-Propeptide of Type II Cartilage Collagen Resulting in Imperfect Fibril Assembly
}

\author{
A. Robin Poole, * I. Pidoux, * A. Reiner, * L. Rosenberg, ${ }^{\text {D. Hollister, }}$ ' L. Murray," and D. Rimoin" \\ *Joint Diseases Laboratory, Shriners Hospital for Crippled Children, Department of Experimental Surgery, McGill University, \\ Montreal, Quebec, H3G 1A6. Canada; ${ }^{\ddagger}$ Orthopedic Research Laboratories, Montefiore Medical Center, Bronx, New York, 10467; \\ ${ }^{\S}$ Research Laboratory, Shriners Hospital for Crippled Children, Portland, Oregon 97201; "Division of Medical Genetics, \\ Department of Pediatrics, Harbor/UCLA Medical Center, Torrance, California 90509
}

\begin{abstract}
Epiphyseal and growth plate cartilages from four cases of Kniest dysplasia have been studied. In each case collagen fibril organization appeared abnormal by electron microscopy compared with age-matched normal cartilages: fibrils were much thinner, of irregular shape and did not exhibit the characteristic banding pattern. This was associated with the absence (compared with normal cartilage) of the $\mathrm{C}$-propeptide of type II collagen (chondrocalcin) from the extracellular matrix of epiphyseal cartilages, although it was detected (as in normal cartilages) in the lower hypertrophic zone of the growth plate in association with calcifying cartilage. The $C$-propeptide was abnormally concentrated in intracellular vacuolar sites in Kniest cartilages and its total content was reduced in all cases but not in all cartilages. Moreover, it was not a part of the procollagen molecule. In contrast, type II collagen $\alpha$-chain size was normal, indicating the formation of a triple helix. Also type II collagen content was normal and it was present in extracellular sites and only occasionally detected intracellularly. These observations suggest that the defect in Kniest dysplasia may result from the secretion of type II procollagen lacking the C-propeptide and abnormal fibril formation, and that the $\mathbf{C}$ propeptide is normally required for fibril formation.
\end{abstract}

\section{Introduction}

The human chondrodysplasias are a heterogeneous group of disorders that result in disproportionate short stature $(1,2)$. Although it has been postulated that these conditions frequently involve abnormalities of cartilage collagen synthesis and organization, there is very little direct confirmed evidence of specific collagen defects. In one case of an unusual spondyloepiphyseal dysplasia, electron microscopy demonstrated focal disorganization of dermal collagen fibers (3), but this has

Address reprint requests to Dr. Poole, Joint Diseases Laboratory, Shriners Hospital for Crippled Children, Department of Experimental Surgery, McGill University, 1529 Cedar Avenue, Montreal, Quebec, H3G 1A6, Canada. Dr. Rimoin's present address is Department of Pediatrics, Cedars-Sinai Medical Center, Los Angeles, CA.

Received for publication 2 December 1986 and in revised form 22 July 1987.

\section{J. Clin. Invest.}

(c) The American Society for Clinical Investigation, Inc.

$0021-9738 / 88 / 02 / 0579 / 11 \quad \$ 2.00$

Volume 81, February 1988, 579-589 not been confirmed in other cases. However, type II collagen chains of higher molecular weight have been found in the cartilages of a series of cases of varying types of spondyloepiphyseal dysplasia (4). In various forms of osteogenesis imperfecta, Ehlers-Danlos syndromes, Marfan syndrome, and cutis laxa, however, there is a considerable body of evidence demonstrating a wide range of abnormalities in collagen processing during synthesis and secretion that are associated with the abnormal biomechanical properties of the tissues containing these molecules (5-7). These changes range from impaired transcription and cross-link formation to decreased secretion and abnormal fibril formation.

Patients with Kniest dysplasia are characterized by short stature, a flat nasal bridge, cleft palate, hearing defects, joint contractures and stiffness, kyphoscoliosis (8), myopia and vitreoretinal degeneration (9). Extensive degeneration of the extracellular matrix in the growth plate and adjacent resting epiphyseal cartilage has been observed (10) and the cartilage has a crumbly consistency. Together these abnormalities are suggestive of a defect involving type II collagen since this molecule is present in hyaline cartilages of all the organs and tissues affected, including the vitreous of the eye (11-13).

Recently we isolated a matrix protein from fetal bovine epiphyseal cartilage (14). We discovered that this protein is intimately associated with developing cartilage and the calcification of cartilage matrix in endochondral ossification (15). Sequence analyses revealed that this molecule, which we called chondrocalcin (15), is in fact the C-propeptide of type II collagen (16). We have used a polyclonal monospecific antiserum to this protein to determine its localization, size, and content in epiphyseal and growth plate cartilages of patients with Kniest dysplasia and aged matched normal cartilages. We now show that there are abnormalities in the distribution, content, and processing of the C-propeptide that are associated with abnormal collagen fibril formation.

\section{Methods}

\section{Patients}

A summary of the four Kniest cases studied and the sites from which cartilages were taken at autopsy or biopsy is provided in Table I. Other cartilages studied are shown in Table I. These were isolated from 'normal' individuals who had no history of cartilage abnormalities. These wer age- and site-matched as well as possible. All tissues were stored frozen at $-20^{\circ}$ to $-70^{\circ} \mathrm{C}$ until examined.

\section{Analyses}

Hydroxyproline. Tissue was hydrolyzed in $6 \mathrm{~N} \mathrm{HCl}$ overnight at $115^{\circ} \mathrm{C}$ under nitrogen. It was then dried in vacuo at room temperature and 
Table I. Patients and Cartilages Examined

\begin{tabular}{|c|c|c|c|}
\hline Group/Age & Sex & Autopsy/Biopsy & Tissue \\
\hline \multicolumn{4}{|l|}{ Kniest } \\
\hline $3 \mathrm{mo}$ & $\mathbf{F}$ & A & $\begin{array}{l}\text { EG } \\
\text { CCJ }\end{array}$ \\
\hline $16 \mathrm{mo}$ & $\mathrm{F}$ & A & $\begin{array}{l}\text { EG } \\
\text { CCJ }\end{array}$ \\
\hline $11 \mathrm{yr}$ & $\mathbf{M}$ & B & IC \\
\hline $12 \mathrm{yr}$ & $F$ & B & IC \\
\hline \multicolumn{4}{|l|}{ Normal } \\
\hline Fetus, 15 wk & $\mathbf{M}$ & A & EG \\
\hline Fetus, 19 wk & $F$ & A & $\mathrm{CCJ}$ \\
\hline Fetus, 28 wk & $\mathrm{F}$ & A & EG \\
\hline Newborn (case 1) & $\mathbf{M}$ & A & $\mathrm{CCJ}$ \\
\hline Newborn (case 2) & $\mathbf{M}$ & A & EG \\
\hline 2 mo (case 1$)$ & $F$ & A & $\mathrm{CCJ}$ \\
\hline 2 mo (case 2) & $\mathbf{F}$ & A & A \\
\hline $6 \mathrm{mo}$ & $\mathrm{F}$ & A & A \\
\hline $3 \mathrm{yr}$ & $\mathbf{F}$ & A & A \\
\hline $5 \mathrm{yr}$ & $\mathbf{M}$ & B & $\mathrm{CCJ}$ \\
\hline 9 yr (case 1$)$ & $\mathbf{M}$ & B & CCJ \\
\hline 9 yr (case 2) & $\mathbf{M}$ & B & $\mathrm{CCJ}$ \\
\hline $13 \mathrm{yr}$ & $\mathrm{F}$ & A & A \\
\hline 14 yr (case 1$)$ & $\mathrm{F}$ & B & IC \\
\hline 14 yr (case 2) & $\mathbf{F}$ & B & CCJ \\
\hline $15 \mathrm{yr}$ & $\mathbf{M}$ & A & A \\
\hline
\end{tabular}

EG, epiphysis and growth plate of long bone; A, articular; CCJ, costochondral junction; IC, iliac crest. M, male; F, female.

redissolved in water. Hydroxyproline was determined by amino acid analysis (D-500 amino acid analyzer; Dionex Corp., Sunnyvale, CA).

Isolation and analysis of type II collagen. Cartilage was finely diced and extracted for $72 \mathrm{~h}$ at $4^{\circ} \mathrm{C}$ with $20 \mathrm{vol}$ of $4 \mathrm{M}$ guanidine hydrochloride containing $0.1 \mathrm{M}$ sodium acetate, $\mathrm{pH} 6.0$, with $1 \mathrm{mM}$ ethylenediamine tetraacetic acid, $10 \mathrm{mM} N$-ethylmaleimide and $1 \mathrm{mM}$ phenylmethylsulfonyl fluoride. The residual cartilage was washed twice with ice-cold water to remove guanidine and then digested for $72 \mathrm{~h}$. at $37^{\circ} \mathrm{C}$ with $5 \mu \mathrm{g}$ pepsin $/ \mathrm{ml}$ per $10 \mathrm{mg}$ of tissue in $0.5 \mathrm{M}$ acetic acid with $0.2 \mathrm{M}$ sodium chloride. Pepsin (porcine gastric) was from Boehringer-Mannheim (Indianapolis, IN). The digest was centrifuged at $12,000 \mathrm{~g}$ for 45 min at $4^{\circ} \mathrm{C}$. The supernatant was recovered and analyzed for type II collagen by SDS-PAGE with $2 \%$ mercaptoethanol in a $4 \%$ acrylamide gel (17) followed by silver staining (18).

Extraction of C-propeptide from cartilage. This was extracted at $4^{\circ} \mathrm{C}$ for $48 \mathrm{~h}$ in 20 vol per tissue wet weight of $4 \mathrm{M}$ guanidine hydrochloride with acetate buffer containing proteinase inhibitors as described previously (19). The extracts were diluted at least tenfold in radioimmunoassay buffer before radioimmunoassay for the C-propeptide. Extracts were also analyzed by polyacrylamide gel electrophoresis and immunoblotting as described below after dialysis for $4 \mathrm{~h}$ against water and then against $0.125 \mathrm{M}$ Tris, $\mathrm{pH} 6.8$ containing $0.1 \%$ SDS.

$P A G E$ of cartilage extracts and immunoblotting for C-propeptide. SDS-PAGE of cartilage extracts in the presence of 5\% mercaptoethanol employed a gradient of 4-20\% polyacrylamide (20). Proteins were electrophoretically transferred in a Western blot to nitrocellulose and stained with the rabbit antiserum to the C-propeptide using peroxi- dase-labeled pig anti-rabbit immunoglobulin in conjunction with diaminobenzidene and hydrogen peroxide (15). Nonimmune rabbit serum was used as a control.

Antibodies. A monospecific rabbit antiserum (R117) to the C-propeptide of bovine type II collagen was prepared and characterized for specificity with an extract of bovine epiphyseal cartilage in the manner described previously $(14,15)$. The mouse monoclonal antibody $\left(\operatorname{IgG}_{2}\right)$ to human helical type II collagen has already been described (21). Pig $F\left(a b^{\prime}\right)_{2}$ derived from an antiserum to the $F\left(a b^{\prime}\right)_{2}$ subunits of mouse IgG, prepared as described for other pig anti-(Fab') $)_{2}$ sera (22), was labeled with fluorescein isothiocyanate as described previously (22) for the pig antibody $F\left(a b^{\prime}\right)_{2}$ to rabbit $F\left(a b^{\prime}\right)_{2}$ subunits of rabbit $\operatorname{IgG}$. $F\left(a b^{\prime}\right)_{2}$ preparations of IgG from antiserum R117 and nonimmune rabbit serum were prepared as before (22). Protein concentrations of pig and rabbit $\mathrm{F}\left(\mathrm{ab} \mathrm{b}^{\prime}\right)_{2}$ and mouse IgG were determined by assuming that 1 $\mathrm{mg} / \mathrm{ml}$ in phosphate buffered saline has an optical density of 1.4 in a 1-cm light path cuvette (22).

Immunohistochemistry. Tissues were frozen sectioned at $6 \mu \mathrm{m}$, fixed in freshly prepared $4 \%$ formaldehyde in phosphate buffered saline for 5 min washed and digested with chondroitinase ABC as described (15). The C-propeptide of type II collagen and helical type II collagen were localized using the indirect method of immunofluorescence essentially as described (15). The monoclonal $\mathrm{IgG}_{2 \mathrm{~b}}$ antibody to helical type II collagen was used at $8 \mu \mathrm{g} / \mathrm{ml}$ in conjunction with purified non-immune IgG2b (Miles Laboratories, Rexdale, Ontario) as a control. Binding was detected by a fluorescein-labeled pig $\operatorname{IgG~} \mathrm{F}\left(\mathrm{ab}^{\prime}\right)_{2}$ anti-mouse $\operatorname{IgG~} F\left(a b^{\prime}\right)_{2}$, used in the manner described for the fluorescein-labeled pig $\mathrm{F}\left(\mathrm{ab}^{\prime}\right)_{2}$ anti-rabbit $\operatorname{IgG} \mathrm{F}\left(\mathrm{ab}^{\prime}\right)_{2}(15)$.

Electron microscopy. Frozen tissue from autopsy or biopsy was used for studies of Kniest and normal cartilages. This was fixed in $2 \%$ glutaraldehyde and osmium tetroxide, embedded in Spurr resin, sectioned and stained with lead citrate and uranyl acetate as before (15). Sections were examined with an electron microscope (Philips 400; Philips Electronics, Toronto, Ontario).

Radioimmunoassay for C-propeptide of type II collagen. Labeling of the C-propeptide with ${ }^{125}$ I and the solution phase competition radioimmunoassay are described elsewhere (23).

\section{Results}

Immunohistochemistry. All cartilages of normal individuals appeared well organized, while those from cases with Kniest dysplasia exhibited a disorganized appearance as described previously (10). Tissue sections were always treated after fixation with chondroitinase $A B C$ to remove chondroitin sulfate that could otherwise mask and prevent the detection of tissue antigens in the extracellular matrix.

Helical type II collagen. All normal cartilages examined contained diffuse extracellular staining for type II collagen. Typically, epiphyseal cartilage of a normal newborn (Fig. 1 a) showed diffuse staining for type II collagen throughout the cartilage matrix. In older normal cartilages (such as at 5 and 14 yr), staining was most intense in the territorial matrix (Fig. 1 b). Since collagen fibrils are seen throughout the territorial and interterritorial matrix, this would indicate that in older specimens the epitope is not so commonly detectable in regions most remote from the cell. In growth plate cartilages, staining was evenly distributed in longitudinal and transverse septa (data not shown). Cartilages of all four cases of Kniest dysplasia stained weakly but fairly uniformly for collagen such as is shown in Fig. $1 c$ and $d$. Stronger territorial staining was not observed in these epiphyseal cartilages. Usually, there was little evidence of any significant intracellular accumulation of col- 

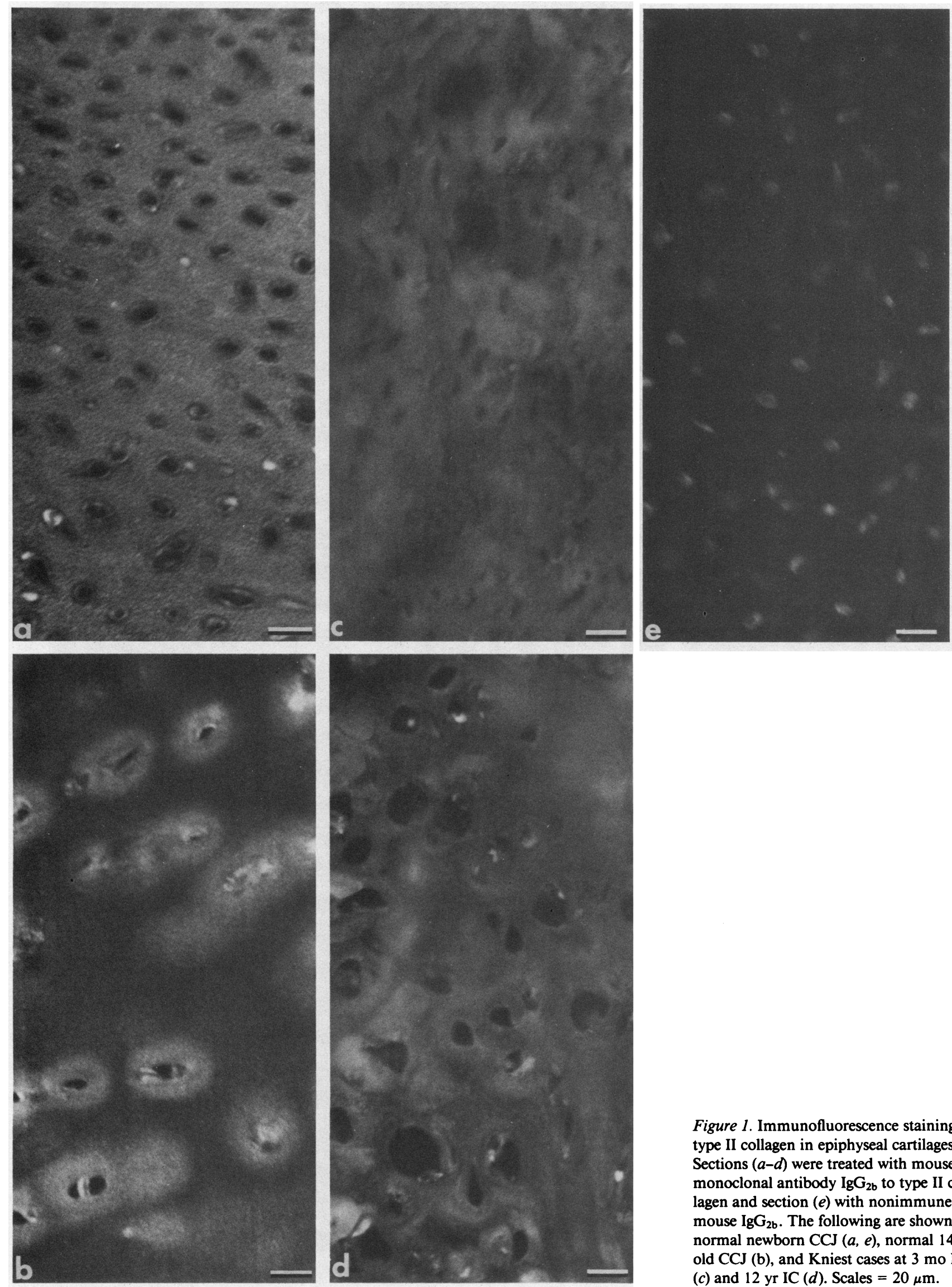

Figure 1. Immunofluorescence staining of type II collagen in epiphyseal cartilages. Sections $(a-d)$ were treated with mouse monoclonal antibody IgG $_{2 b}$ to type II collagen and section $(e)$ with nonimmune mouse $\mathrm{IgG}_{2 \mathrm{~b}}$. The following are shown: a normal newborn $\mathrm{CCJ}(a, e)$, normal 14-yr old CCJ (b), and Kniest cases at 3 mo EG (c) and $12 \mathrm{yr} \mathrm{IC}(d)$. Scales $=20 \mu \mathrm{m}$. 



Figure 2. Immunofluorescence staining of C-propeptide in epiphyseal cartilages. Sections $(a-d, f)$ were treated with rabbit Fab' to C-propeptide and section $(e)$ with nonimmune rabbit Fab'. The following are shown: a normal newborn $\mathrm{CCJ}(a, e)$, normal $14 \mathrm{yr}$ old $\mathrm{CCJ}(b)$, $\mathrm{Kniest}$ cases at 3 mo EG $(c), 16$ mo EG $(f)$, and $12 \mathrm{yr}$ IC $(d)$. Scales $=20 \mu \mathrm{m}$. 
lagen in normals or Kniests although this was occasionally observed in some cells in normal epiphyseal fetal and newborn cartilages (Fig. 1 a). Sections first treated with the control nonimmune $\operatorname{IgG}_{2 b}$ did not stain (Fig. $1 e$ ).

$C$-propeptide. In sections of all cartilages examined from normal individuals such as a normal newborn (Fig. $2 a$ ), staining was seen in the extracellular matrix: in this and in other fetal and newborns case it was evenly distributed and there was some cell-associated diffuse staining. In older cartilages such as the normal 14-yr old, staining was primarily observed in the territorial matrix (Fig. $2 b$ ), as was observed for type II collagen: little intracellular staining was seen. In contrast, little or no extracellular staining was seen in the four Kniest cases (Fig. $2 c, d$, and $f$ ) except in the calcifying lower hypertrophic zone of the growth plate (Fig. $3 b$ ) where the characteristic intense extracellular and cellular staining seen in all normal growth plates examined previously (15) and in the present study (Fig. $3 a$ ) was observed at the bottom of the hypertrophic zone. In both normal and Kniest growth plate cartilages intracellular staining was clearly detected (Fig. $3 a$ and $b$ ). Throughout all
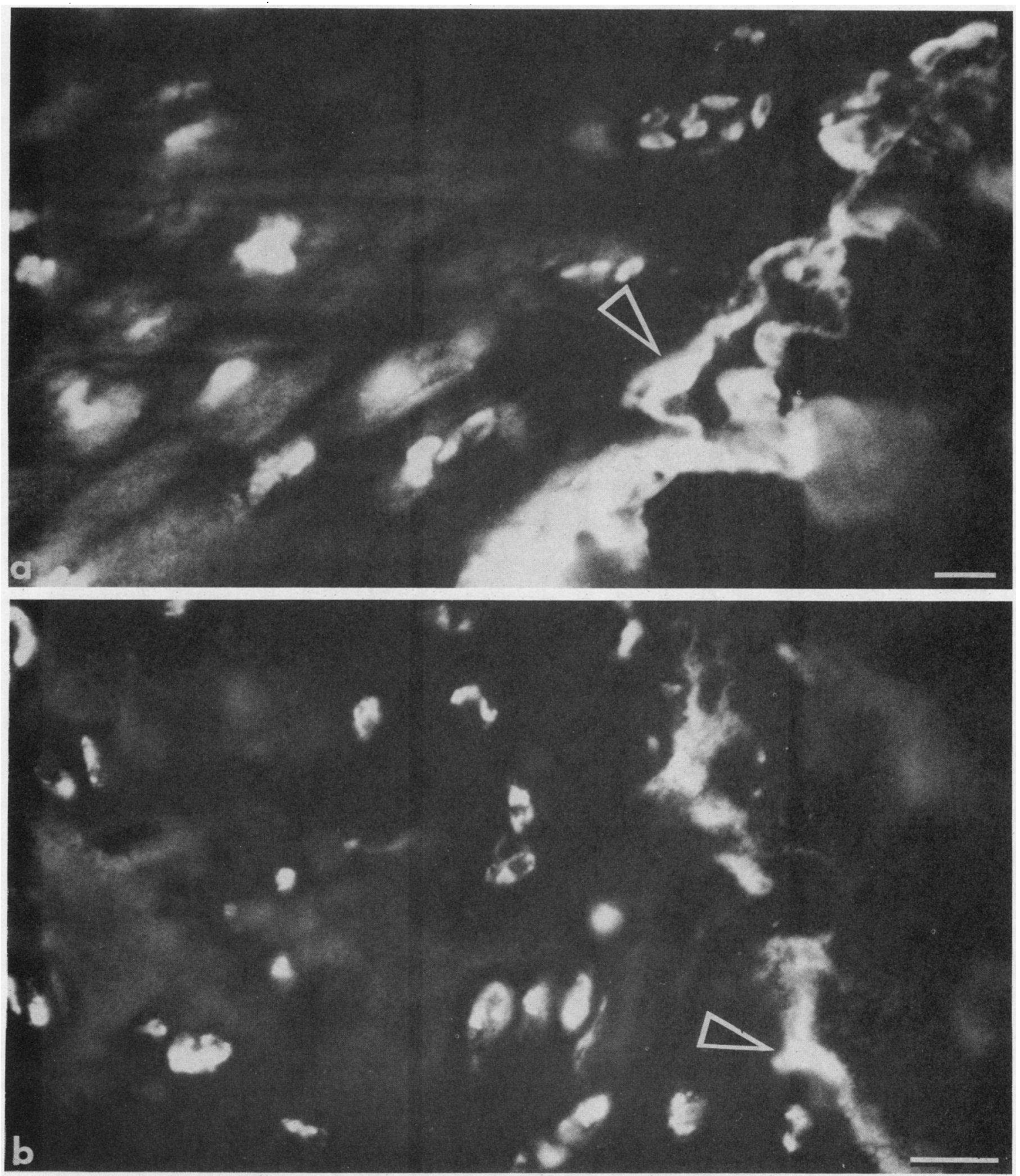

Figure 3. Immunofluorescence of C-propeptide in growth plate cartilages of a normal $14 \mathrm{yr}$ old CCJ (a) and Kniest case at $12 \mathrm{yr}$ IC (b). Arrowheads indicate intense extracellular staining in the lower hypertrophic zone. Scales $=20 \mu \mathrm{m}$. 

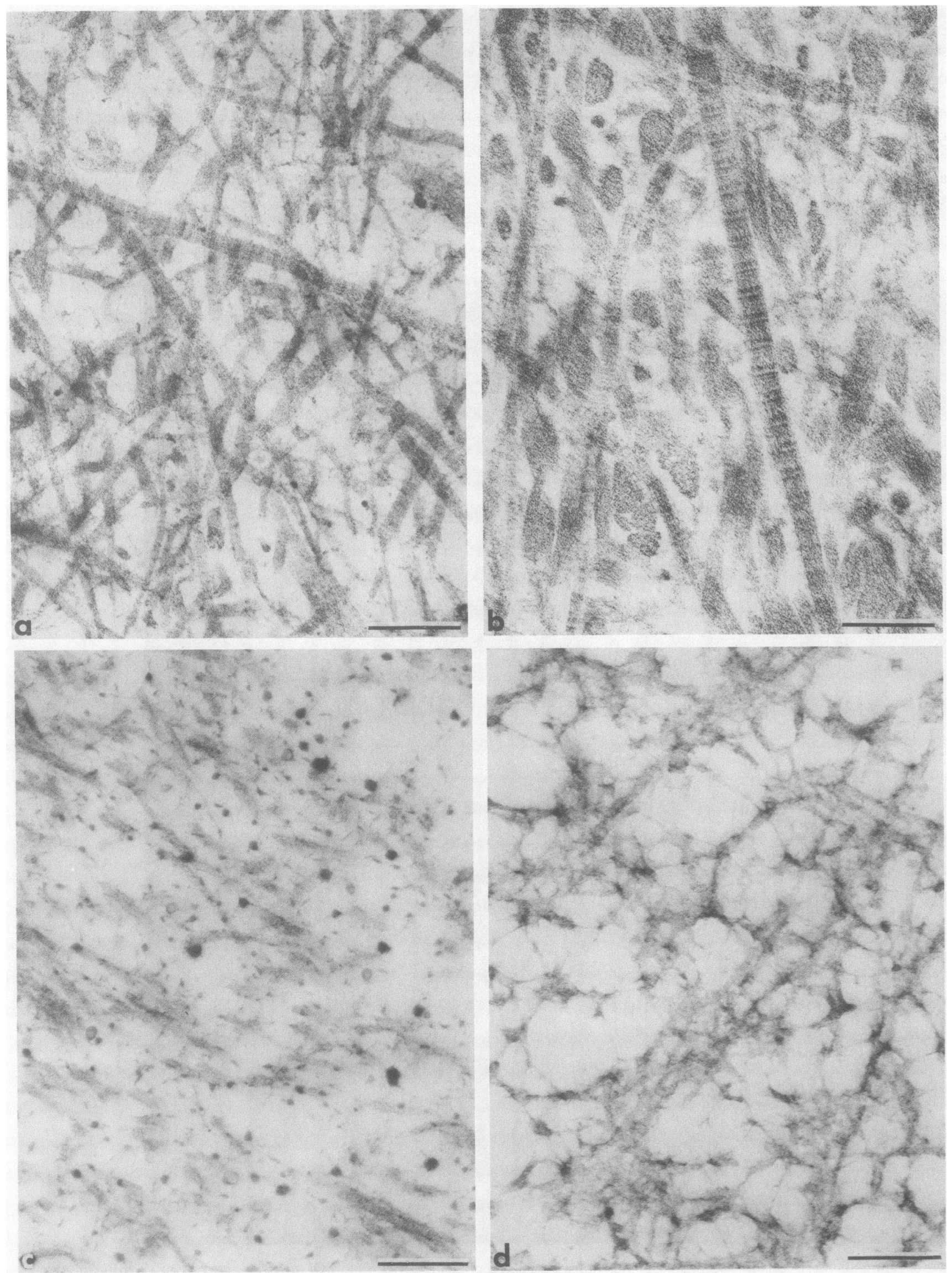

Figure 4. Electron micrographs of epiphyseal cartilages. Normal newborn CCJ (a) and 14-yr old CCJ (b) and Kniest cases at 3 mo EG (c) and $12 \mathrm{yr}$ IC $(d)$. Scales $=200 \mathrm{~nm}$. 
the epiphyseal cartilages and the majority of the growth plate cartilage, staining was present in all cells of Kniest cases and was confined to large intracellular vacuolar sites often of irregular shape (Fig. $2 c, d$, and $f$ ): this staining was intense and well defined. Such intense "vacuolar" intracellular staining for the C-propeptide was never observed in normal nongrowth plate cartilages. Faint cellular staining was observed in sections treated with control non-immune rabbit Fab' (Fig. $2 e$ ).

Electron microscopy. Examination of the resting epiphyseal cartilages of a normal newborn fetus (Fig. $4 a$ ) and 2-mo old revealed the presence of well defined banded fibrils of variable diameter. In an older normal 14-yr old, the epiphyseal cartilage contained banded fibrils of larger diameter that appeared more closely packed (Fig. 4 b). In all normal cartilages that have been studied, fibrils were well defined and showed clear banding patterns. In contrast, this was not seen in Kniest cartilages. The 3-mo old Kniest specimen (Fig. $4 c$ ) displayed a lack of well organized collagen fibrils: instead they were very thin and of irregular shape and appearance and lacked any clear banding pattern. Another 12-yr-old Kniest specimen (Fig. $4 d$ ) contained very thin fibrils, was less well stained and even more irregular in appearance than those normally seen at this age and with no clear banding. Less extreme abnormalities were observed in the other Kniest cases. Thus in Kniest cartilages fibrils were generally thinner than in even the newborn cartilage, much less well stained, without a clear banding pattern and appeared much less "compact" than those seen in specimens of similar age and site.

$C$-propeptide and hydroxyproline contents. These results are shown in Table II. In normal tissues the C-propeptide content was generally at its highest in newborn cartilages and dropped after birth with two exceptions in a total of 13 specimens from 11 cases. After birth, hydroxyproline contents of normal tissues usually increased after 6 mo reaching approximately twice the concentration at birth. In most cases, the molar ratio of collagen to C-propeptide increased after birth as C-propeptide levels dropped and collagen contents increased. Individual cartilages as well as specimens from persons of similar ages, however, exhibited significant differences in contents of C-propeptide and hydroxyproline. It can be seen that in the two specimens of the 3-mo old Kniest, the C-propeptide content was higher in epiphyseal-growth cartilage compared with the relatively small amount present in the costochondral junction. In the former case the molar ratio of collagen to C-pro-

Table II. C-Propeptide and Collagen Contents of Epiphyseal and Growth Plate Cartilages from Normal Controls and Those with Kniest Dysplasia

\begin{tabular}{|c|c|c|c|c|}
\hline $\begin{array}{l}\text { Specimen } \\
\text { normal/Kniest (N/K) } \\
\text { age }\end{array}$ & Tissue & C-propeptide & Hydroxyproline & $\begin{array}{l}\text { Molar ratio } \\
\text { collagen/C-propeptide }\end{array}$ \\
\hline & & $n g / m g$ wet wt & $\mu g / m g$ wet $w t$ & \\
\hline N Newborn (case 1) & $\mathrm{CCJ}-1$ & 143.0 & 10.3 & 225 \\
\hline N Newborn (case 1) & $\mathrm{CCJ}-2$ & 77.0 & 11.4 & 463 \\
\hline N Newborn (case 2) & EG & 72.0 & 10.9 & 473 \\
\hline N 2 mo (case 1) & CCJ & 11.0 & 11.2 & 3,180 \\
\hline N 2 mo (case 2) & A & 15.0 & 18.0 & 3,749 \\
\hline K 3 mo & EG & 47.0 & 8.5 & 565 \\
\hline K 3 mo & CCJ & 5.0 & 9.9 & 6,189 \\
\hline N 6 mo & A & 19.5 & 10.8 & 1,731 \\
\hline K $16 \mathrm{mo}$ & EG & 7.0 & 20.9 & 9,326 \\
\hline K $16 \mathrm{mo}$ & $\mathrm{CCJ}$ & 5.9 & 5.7 & 3,019 \\
\hline $\mathrm{N} 3 \mathrm{yr}$ & A & 25.0 & 19.1 & 2,388 \\
\hline $\mathrm{N} 9$ yr (case 1) & $\mathrm{CCJ}$ & 22.3 & 11.4 & 1,597 \\
\hline N 9 yr (case 2) & CCJ & 83.0 & 11.4 & 430 \\
\hline $\mathrm{K} 12 \mathrm{yr}$ & IC-1 & 23.0 & 18.7 & 2,541 \\
\hline $\mathrm{K} 12 \mathrm{yr}$ & IC-2 & 7.1 & 13.4 & 5,899 \\
\hline N 13 yr & A & 3.0 & 19.0 & 19,772 \\
\hline N 14 yr (case 1$)$ & IC & 34.0 & 8.2 & 754 \\
\hline N 14 yr (case 1$)$ & CCJ & 98.0 & 17.2 & 549 \\
\hline $\mathrm{N} 15 \mathrm{yr}$ & A & 13.0 & 24.8 & 5,962 \\
\hline
\end{tabular}

CCJ, costochondral; EG, epiphysis/growth plate; IC, iliac crest; ND, not determined. The molecular weight of collagen was determined from its known amino acid composition. The molecular weight used for the C-propeptide was 105,000 (42). Molecular weight of helical collagen without the propeptide was taken as 300,000 based on its amino acid composition (43). Hydroxyproline was determined as representing $11.2 \%$ of the total weight of helical collagen. 
peptide was within the normal range observed at birth and in the latter specimen was lower than levels seen at 2 mo in comparable tissue. The specimens from the costochondral cartilage of the 16-mo old Kniest contained similar amounts of C-propeptide to that seen in this tissue in the normal at 2 mo but less than that seen in two cases at 9 yr. The other Kniest specimen of epiphyseal growth plate cartilage also contained much less C-propeptide compared with that seen in the newborn and in normal articular cartilages at 6 mo and $3 \mathrm{yr}$. Kniest specimens of the iliac crest at $12 \mathrm{yr}$ displayed lower C-propeptide contents compared with normal iliac crest at 14 yr although hydroxyproline levels here and in most Kniest cartilages were within normal ranges seen in other tissues. The molar ratios of collagen to C-propeptide were clearly elevated in both Kniest specimens at $12 \mathrm{yr}$, one at $16 \mathrm{mo}$ and one specimen at 3 mo compared with the values for the same but normal tissues and other normal tissues of comparable age. Overall, therefore, there was an indication that there was a reduction in C-propeptide content compared with collagen in all Kniest cases but not in all cartilages from the same individual.

SDS-PAGE and immunoblotting analyses. SDS-PAGE analyses of type II collagen isolated from three Kniest cartilages (tracks 1-3) shown in Figs. 1 and 2 and one typical normal cartilage (track 4 ) revealed that the collagen $\alpha$-chains are of normal size in the Kniest specimens examined (Fig. 5). There was more of the $\gamma$-component in Kniest cases at 3 and 16 mo than is normally observed in comparable cartilages from normal cases (L. Murray, unpublished). Whether this represents an increased formation of cross-links remains to be established.

Immunoblots (Western blots) revealed that in both normal and Kniest cartilages, the majority of the C-propeptide is not a part of the procollagen molecule (see below) and has a subunit

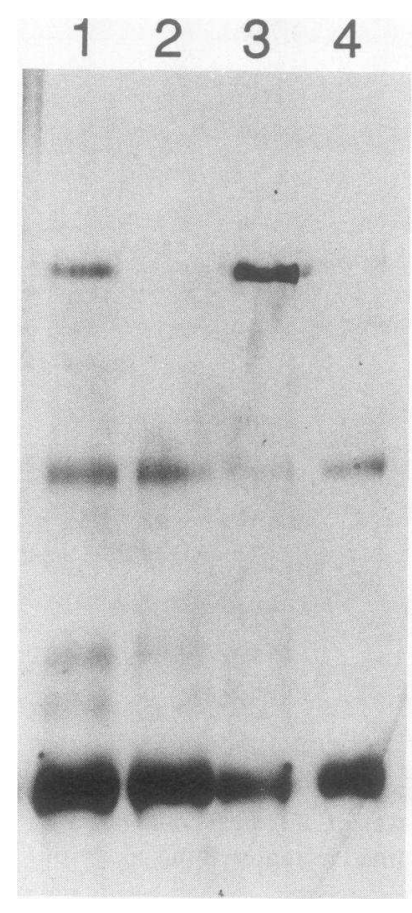

Figure 5. Analyses of type II collagen $\alpha$ chain sizes of normal and Kniest cartilages. The $\gamma, \beta$, and $\alpha$ components are indicated. Track 1,2 and 3 contain collagen of Kniest cartilages at $16 \mathrm{mo}(\mathrm{EG}), 12 \mathrm{yr}$ (IC), and 3 mo (EG), re$\alpha$ spectively. Track 4 contains the type II collagen from a normal 19-wk fetus (CCJ). of $M_{\mathrm{r}}=35,000$ (Fig. 6) comparable in size to that reported for fetal bovine epiphyseal cartilage $(14,15)$. The slightly smaller subunit seen in bovine cartilage (15) was observed in the normal cartilage and in two of three Kniest cases. The reduced staining observed for the 16-mo old Kniest reflects the much lower content of C-propeptide present in this specimen (see Table II). Other lower molecular weight immunoreactive species are probably degradation products produced before autopsy and freezing of the samples: these are not normally seen in freshly isolated bovine cartilages. Weakly stained bands seen in the region close to $M_{\mathrm{r}}=54,000$ represent nonspecific staining since they are clearly seen in the preimmune control (Fig. 6 , left side). Higher molecular weight species were detected in trace amounts (as faint staining) in the region of $M_{\mathrm{r}}=153,000$, 143,000 , and 133,000 (data not clearly visible on the photomicrograph). These are considered to represent immunoreactive procollagen containing the $\mathrm{C}$-propeptide since studies of bovine cartilage have demonstrated the susceptibility of immunoreactive molecules of this size to bacterial collagenase (Poole, A. R., and A. Reiner, to be submitted).

\section{Discussion}

This study provide some insight into the reasons for the abnormal development and properties of hyaline cartilages in Kniest dysplasia. Normal and Kniest tissues were age and site matched as well as possible. The results we show are typical of those found in these cartilages. There was no evidence that the abnormalities we detected in Kniest cartilages were restricted to any particular uncalcified cartilage nor were they seen only at a particular age. These differences were never seen in normal cartilages. With electron microscopy the collagen fibrils look abnormal in Kniest dysplasia suggesting abnormal fibril assembly. This may be explained as follows. The intracellular formation of the triple helix of procollagen is thought to proceed from the C-terminal end of the molecule, starting with the association of the newly synthesized pro $\alpha$-chains as the Cpropeptides interact and interchain disulfide bond formation occurs (24-28). That helix formation occurs in Kniest dysplasia is indicated by the normal $\alpha$-chain length of the type II collagen: if a triple helix had not formed, digestion of collagen with pepsin would have resulted in cleavage of the $\alpha$-chains. Ordinarily, the $\mathrm{C}$-propeptide, together with the $\mathrm{N}$-propeptide, are thought to be removed from the procollagen molecule (to give tropocollagen) at the cell surface or, at least, in an extracellular site $(29,30)$. These events are normally catalyzed by specific $\mathrm{N}$ and C-propeptidases (30). In Kniest dysplasia, in the epiphyseal cartilages outside the growth plate, the C-propeptide does not appear to be secreted: if it is secreted then it is in considerably reduced amounts. It accumulates within large vacuolar dilatations within the chondrocytes, as indicated by our immunofluorescence studies. Our analytical studies suggest that the majority of the C-propeptide was cleaved from procollagen within these cells. The quantitative deficiency of C-propeptide that was observed in the three cases examined but not in all specimens may result in part from the lack of secretion and accumulation of this species within the extracellular matrix, as is normally observed. Since the helical collagen 


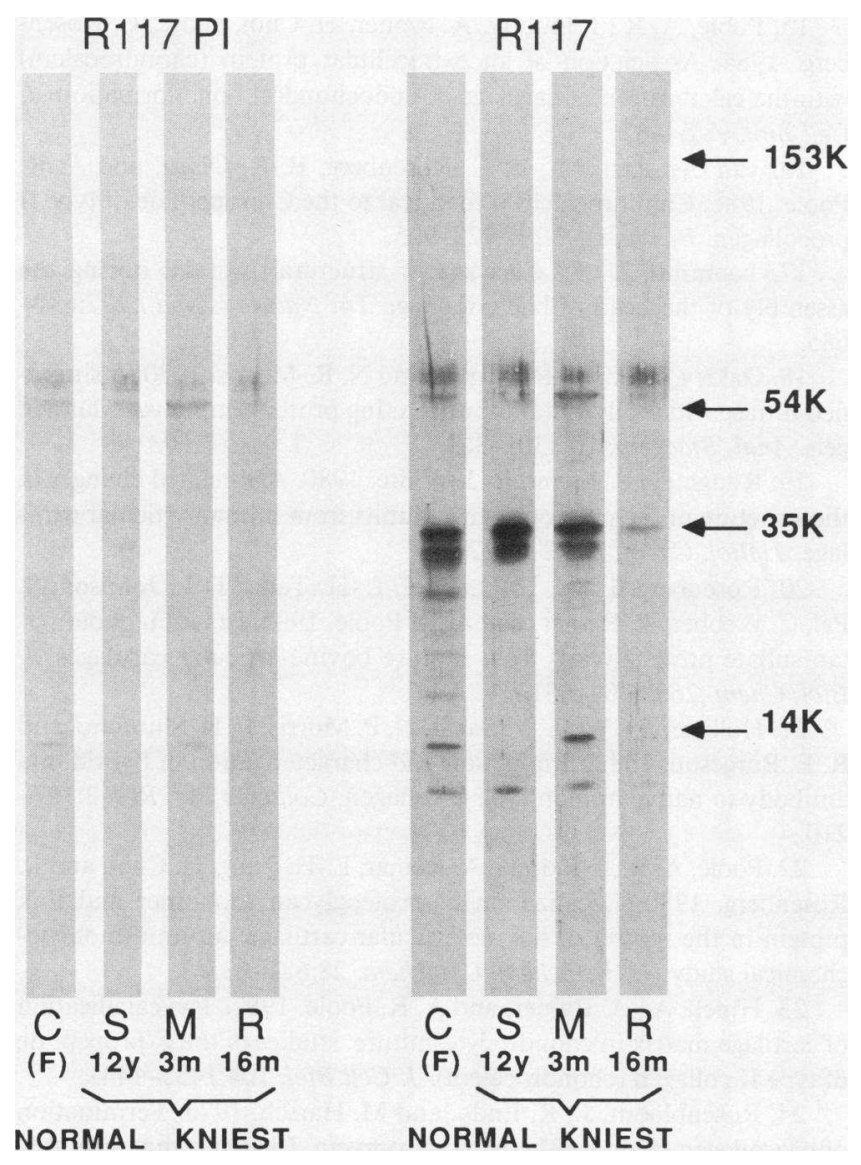

Figure 6. Analyses of normal and Kniest cartilage extracts for C-propeptide by SDS-PAGE (gradient gel 4-20\%) and Western blotting to nitrocellulose followed by staining with antiserum (R117) to C-propeptide of type II collagen. Preimmune serum (R117 PI) controls are also shown. The normal (C) was a 28-wk old fetus EG. Each track contains 10-30 $\mu \mathrm{l}$ of sample representing the extract from $1 \mathrm{mg}$ of tissue. $M_{\mathrm{r}}$ are indicated where $\mathrm{K}=1,000$.

content and distribution in the Kniest cartilage was not detectably abnormal, as indicated by hydroxyproline analyses and immunofluorescence, it would appear that even in normal cartilage a significant proportion of C-propeptide cannot be accounted for if one assumes that there are equimolar amounts of C-propeptide and collagen. Hence significant extracellular and intracellular degradation and/or loss of the Cpropeptide may have occurred in normal and Kniest cartilages, respectively.

Thus it would appear that procollagen is synthesized normally in Kniest dysplasia but the C-propeptide may be prematurely removed within the cell. The absence of the C-propeptide from secreted procollagen may then account for the imperfect fibril assembly which we have observed. Earlier studies have suggested that the $\mathrm{C}$-propeptide, and the $\mathrm{N}$-propeptide, and their subsequent removal are required for normal fibril assembly (31-35), particularly since there appears to be an obligatory order for the removal of the two propeptides from the procollagen molecule in the formation of the fiber (30). Hence the propeptides may provide a specific registration of procollagen in the newly forming fibril. Our studies would support this belief and indicate the importance of the C-propeptide in this process.

It may also be argued that the C-propeptide was never synthesised as part of the procollagen molecule and hence that the C-propeptide is not required for the assembly of the triple helix. This is considered unlikely in view of the normal $\alpha$ chain length and the implication of this observation as discussed above.

Cleavage of the C-propeptide within the cell in Kniest dysplasia may occur within the endoplasmic reticulum since large dilatations of this organellar network have been observed in chondrocytes from individuals with Kniest dysplasia $(8,36)$ and the vacuolar localization of the $\mathrm{C}$-propeptide is consistent with an intracellular distribution of this kind. This is to be investigated further using immunoelectron microscopy. The accumulation of the $C$-propeptide in the cell also suggests that for it to be translocated and secreted it must be a part of the procollagen molecule. The nature of the C-propeptidase responsible for this putative, premature cleavage is not known although it is interesting to note that the enzyme which is normally responsible for the extracellular cleavage has been reported to have the properties of both a metallo-protease and an aspartate protease (37). Thus it resembles, in part, cathepsin $\mathrm{D}$ an intracellular lysosomal aspartate protease which is normally active at acid $\mathrm{pH}$. It is possible that there exists within the Kniest chondrocyte an intracellular compartment associated with the endoplasmic reticulum where the removal of the C-propeptide occurs prematurely: this may be mediated either by the C-propeptidase or by a protease with similar or the same cleavage specificity, which could include cathespin D and related species as suggested by others in studies of procollagen processing $(38,39)$. The apparent intracellular removal of the C-propeptide from procollagen also indicates that the proteinase(s) responsible for this event is abnormally active within the cell. In addition, the abnormal accumulation of C-propeptide within these cells may secondarily adversely affect other biosynthetic and secretory mechanisms leading to other changes in cartilage matrix and cellular physiology.

The relatively normal extracellular accumulation of the C-propeptide in the lower hypertrophic zone in growth plate cartilages of those with Kniest dysplasia, as we have previously described in association with calcification of the cartilage matrix (15), indicates that the mechanism(s) responsible for the major release of the $\mathrm{C}$-propeptide in calcifying cartilage and its regulation are different to those found in more immature hypertrophic chondrocytes and in other chondrocytes. Evidence for this has also been obtained from in vitro studies of isolated chondrocytes (23). The more normal C-propeptide contents observed in some of the Kniest specimens may result from the inclusion of a significant proportion of growth plate cartilage in these specimens.

Finally, to place these observations in perspective, it is worth noting that there have been few reports of abnormalities in the processing of procollagen. Originally, in cattle with the condition called dermatosparaxis, it was observed that the amino-propeptidase capable of cleaving type 1 procollagen could not be detected (40). In man, there is an impairment or lack of removal of the amino-propeptide of type I collagen in Ehlers-Danlos Syndrome type VII and in a variant of osteo- 
genesis imperfecta (30): in the latter case this is associated with a deletion of the pro $\alpha 2$ (1) chain. Recently, nonexpression of type II collagen in a case of Langer-Saldino achondrogenesis was reported (41). Other than this, Kniest dysplasia is the only human condition we know of where there is an apparent abnormality in the type II collagen. The myopia and vitroretinal degeneration which are observed in this condition (9) may also result from an abnormality of type II collagen processing in the posterior chamber of the eye.

\section{Acknowledgments}

We thank Freda Rowbotham and Michele Burman Turner for processing this manuscript and Mark Lepik for some of the artwork.

This work was funded by the Shriners of North America (to ARP and $\mathrm{DH}$ ) and the National Institutes of Health (to LR, LM, and DR).

\section{References}

1. Sillence, D. O., W. A. Horton, and D. L. Rimoin. 1979. Morphologic studies in the skeletal dysplasias. A review. Am. J. Pathol. 96:811-870.

2. Rimoin, D. L., and R. S. Lachman. 1983. The chondrodysplasias. In Principles and Practice of Medical Genetics. Vol. 2. A. E. H. Emery and D. L. Rimoin, editors, Churchill Livingstone, New York. 703-735.

3. Byers, P. H., K. A. Holbrook, J. G. Hall, P. Bornstein, and J. W. Chandler. 1978. A new variety of spondyloepiphyseal dysplasia characterized by punctate corneal dystrophy and abnormal dermal collagen fibrils. Hum. Genet. 40:157-169.

4. Murray, L. W., and D. L. Rimoin. 1985 Type II collagen abnormalities in the spondylo-epi metaphyseal dysplasias. Am. J. Hum. Gen. 37:A13.

5. Hollister, D. W., P. H. Byers, and K. A. Holbrook. 1982. Genetic disorders of collagen metabolism. Advances in Human Genetics. Vol. 12. H. Harris and K. Hirschhorn, editors. Plenum, New York. pp. $1-87$.

6. Prockop, D. J., and K. I. Kivirikko. 1984. Heritable diseases of collagen. N. Engl. J. Med. 311:376-386.

7. Cheah, K. S. E. 1985. Collagen genes and inherited connective tissue disease. Biochem. J. 229:287-303.

8. Siggers, D. C., D. L. Rimoin, J. P. Dorst, S. B. Dotz, B. R. Williams, D. W. Hollister, R. Silberberg, R. E. Cranley, R. L. Kaufman, and V. A. McKusick. 1974. The kniest syndrome. Birth Defects. 10:193-208.

9. Maumenee, I. H., and E. I. Traboulsi. 1985. The ocular findings in Kniest dysplasia. Am. J. Ophthal. 100:155-160.

10. Horton, W. A., and D. L. Rimoin. 1970. Kniest dysplasia. A histochemical study of the growth plate. Pediatr. Res. 13:1266-1270.

11. von der Mark, K., H. von der Mark, R. Timpl, and R. L. Trelstad. 1977. Immunofluorescent localization of collagen types I, II and III in the embryonic chick eye. Dev. Biol. 59:75-85.

12. Snowden, J. M., and D. A. Swann. 1980. Vitreous structure, V. The morphology and thermal stability of vitreous collagen fibers and comparison to articular cartilage (type II) collagen. Ophthal. Vis. Sci. 19:610-618.

13. Swann, D. A., and S. S. Sotman. 1980. The chemical composition of bovine vitreous-humour collagen fibres. Biochem. J. 185:545554.

14. Choi, H. U., L.-H. Tang, T. L. Johnson, S. Pal, L. C. Rosenberg, A. Reiner, and A. R. Poole. 1983. Isolation and characterization of a 35,000 molecular weight subunit fetal cartilage matrix protein. $J$. Biol. Chem. 258:655-661.
15. Poole, A. R., I. Pidoux, A. Reiner, H. Choi, and L. C. Rosenberg. 1984. Association of an extracellular protein (chondrocalcin) with the calcification of cartilage in endochondral bone formation. $J$. Cell Biol. 98:54-65.

16. van der Rest, M., L. C. Rosenberg, B. R. Olsen, and A. R. Poole. 1986. Chondrocalcin is identical to the C-propeptide of type II procollagen. Biochem. J. 297:923-925.

17. Laemmli, U. K. Cleavage of structural proteins during the assembly of the head of bacteriophage T4. Nature (Lond.) 227:680685.

18. Oakley, B. R., D. R. Kirsch, and N. R. Morris. 1980. A simplified ultrasensitive silver stain for detecting proteins in polyacrylamide gels. Anal. Biochem. 105:361-363.

19. Roughley, P. J., and R. J. White. 1980. Age-related changes in the structure of the proteoglycan subunits from human articular cartilage. J. Biol. Chem. 255:217-224.

20. Rosenberg, L. C., H. U. Choi, L.-H. Tang, T. L. Johnson, S. Pal, C. Webber, A. Reiner, and A. R. Poole. 1985. Isolation of dermatan sulfate proteoglycans from mature bovine articular cartilages. $J$. Biol. Chem. 260:6304-6313.

21. Hollister, D. W., L. Y. Sakai, N. P. Morris, L. H. Shimono, and R. E. Burgeson. 1982. Production and characterization of hybridoma antibody to native human type II collagen. Collagen Rel. Res. 2:197210.

22. Poole, A. R., I. Pidoux, A. Reiner, L.-H. Tang, H. Choi, and L. Rosenberg. 1980. Localization of proteoglycan monomer and link protein in the matrix of bovine articular cartilage: an immunohistochemical study. J. Histochem. Cytochem. 28:621-635.

23. Hinek, A., A. Reiner, and A. R. Poole. 1987. The calcification of cartilage matrix in chondrocyte culture: studies of the C-propeptide of type II collagen (chondrocalcin). J. Cell Biol. 104:1435-1441.

24. Rosenbloom, J., R. Endo, and M. Harsch. 1976. Termination of procollagen chain synthesis by puromycin. Evidence that assembly and secretion require a $\mathrm{COOH}$ terminal extension. J. Biol. Chem. 251:2070-2076.

25. Bächinger, H. P., L. I. Fessler, R. Timpl, and J. H. Fessler. 1981. Chain assembly intermediate in the biosynthesis of type III procollagen in chick embryo blood vessels. J. Biol. Chem. 256:1319313199.

26. Bruckner, P., E. F. Eikenberry, and D. J. Prockop. 1981. Formation of the triple helix of type I procollagen in cellulo. A kinetic model based on cis-trans isomerization of peptide bonds. Eur. J. Biochem. 118:607-613.

27. Schofield, J. D., J. Uitto, and D. J. Prockop. 1974. Formation of interchain disulfide bonds and helical structure during biosynthesis of procollagen by embryonic tendon cells. Biochemistry. 13:18011806.

28. Uitto, J., and D. J. Prockop. 1974. Biosynthesis of cartilage procollagen. Influence of chain association and hydroxylation of prolyl residues on the folding of the polypeptides into the triple-helical conformation. Biochemistry. 13:4586-4591.

29. Prockop, D. J., K. I. Kivirikko, L. Tuderman, and N. A. Guzman. 1979. The biosynthesis of collagen and its disorders. $N$. Engl. J. Med. 301:13-23; 77-85.

30. Peltonen, L., R. Halila, and L. Ryhänen. 1985. Enzymes converting procollagens to collagens. J. Cell. Biochem. 28:15-21.

31. Miyahara, N., F. K. Njieha, and D. J. Prockop. 1982. Formation of collagen fibrils in vitro by cleavage of procollagen with procollagen proteinases. J. Biol. Chem. 257:8442-8448.

32. Uitto, J., R. E. Allan, and K. L. Polak. 1979. Conversion of type II procollagen to collagen. Extracellular removal of the amino-terminal and carboxy-terminal extensions without a preferential sequence. Eur. J. Biochem. 99:97-103.

33. Fleischmajer, R., R. Timpl, L. Tuderman, L. Raisher, M. Wiestner, J. S. Perlish, and P. N. Graves. 1981. Ultrastructural identification of extension aminopropeptides of type I and III collagens in human skin. Proc. Natl. Acad. Sci. USA. 78:7360-7364. 
34. Fleischmajer, R., B. R. Olsen, R. Timpl, J. S. Perlish, and O. Lovelace. 1983. Collagen fibril formation during embryogenesis. Proc. Natl. Acad. Sci. USA. 80:3354-3358.

35. Miyahara, M., P. Bruckner, O. Hello, and D. J. Prockop. 1983. Aggregation of a type I collagen precursor containing N-terminal propeptides. Coll. Rel. Res. 3:279-291.

36. Stanescu, V., R. Stanescu, and P. Maroteaux. 1984. Pathogenic mechanisms in osteochondrodysplasias. J. Bone Jt. Surg. 66-A:817836.

37. Hojima, Y., M. van der Rest, and D. J. Prockop. 1985. Type I procollagen carboxyl-terminal from chick embryo tendons. Purification and characterization. J. Biol. Chem. 260:15996-16003.

38. Davidson, J. M., L. S. G. McEneany, and P. Bornstein. 1979. Procollagen processing. Limited proteolysis of $\mathrm{COOH}$-terminal extension peptides by a cathepsin-like protease secreted by tendon fibroblasts. Eur. J. Biochem. 100:551-558.
39. Helseth, D. J., Jr., and A. Veis. 1984. Cathespin D-mediated processing of procollagen: lysosomal enzyme involvement in secretory processing of procollagen. Proc. Natl. Acad. Sci. USA. 81:3302-3306.

40. Lapiere, C. M., A. Lenaers, and L. D. Kohn. 1971. Procollagen peptidase: an enzyme excising the coordination peptides of procollagen. Proc. Natl. Acad. Sci. USA. 68:3054-3058.

41. Eyre, D. R., M. P. Upton, F. D. Shapiro, R. H. Wilkinson, and G. F. Vawter. 1986. Nonexpression of cartilage type II collagen in a case of Langer-Saldino achondrogenesis. Am. J. Hum. Genet. 39:5267.

42. Curran, S., and D. J. Prockop. 1984. Isolation and partial characterization of the carboxyterminal propeptide of type II procollagen from chick embryos. Biochemistry. 23:741-746.

43. Miller, E. J., and L. G. Lunde. 1973. Isolation and characterization of the cyanogen bromide peptides from the $\alpha 1$ (II) chain of bovine and human cartilage collagen. Biochemistry. 12:3153-3159. 